\title{
Risk Assessment of Critical Time to Renewable Operation with Steady-state Security Region
}

\author{
Yunhe Hou \\ The University of Hong Kong \\ Hong Kong, China \\ yhhou@eee.hku.hk
}

\author{
Jie Yan \\ Economic Study Group, MISO \\ Saint Paul, MN, USA \\ jyan@misoenergy.org
}

\author{
Chaoyi Peng, Zhijun Qin, \\ Shunbo Lei \\ The University of Hong Kong \\ Hong Kong, China \\ zjqin@eee.hku.hk
}

\author{
Haiming Ruan \\ Clou Electronics Co., Ltd \\ Shenzhen, China \\ rhm2009@gmail.com
}

\begin{abstract}
Uncertain and variable characteristics of renewable energy resources introduce challenges to power system operation. A normal operating point might be drifted towards an unreliable operating point due to stochastic outputs od renewables. This paper proposes a novel method for estimating critical time to unreliable operating point with steady-state constraints. In this work, a stochastic differential equation is employed to describe the distribution of renewables with predictable tendency and stochastic errors of prediction; meanwhile, the DC power flow based steady-state security region is used to restrict the injected space. To find the critical time that uncontrollable renewables leave the security region, according the flexibility requirements defined by NERC, the uncontrollable region is identified with the Fourier-Motzkin elimination first. And then, by solving the Chebychev center problem, the critical distance for variable renewable outputs is obtained. Finally, an analytical solution of expected exit-time for renewable outputs leaving the security region is given with the Martingale stopping theorem. The proposed method can be used to construct the condition-driven risk indicators. An illustrative example is employed to demonstrate and validate the proposed method.
\end{abstract}

Index Terms-Renewables integration, flexibility requirements, steady-state security region, Fourier-Motzkin elimination, Chebychev center, first hitting time

\section{INTRODUCTION}

Energy crisis and global warming concerns are promoting the governments all over the world to integrate more renewables into power grids. Currently, to promote sustainable energy resources and reduce carbon emissions, the massive penetration of renewable, such as wind and solar power, has been integrating into power systems throughout the world. Associated with tremendous benefit of renewables, essential uncertainty and variability significantly challenge the current power system operating methodology, which balances the highly predictable loads with highly controllable generators. The North American Electric Reliability Corporation (NERC) Integration of Variable Generation Task Force (IVGTF) has identified that a series of challenges of power system operation and planning for variable generation integration by a special report [1]. It is suggested that due to the increase in the overall variability, the penetration of variable generation reaches relatively high levels, the characteristics and operation of the bulk power system will be significantly altered. This critical requirement covers both system operation and planning. In the new energy paradigm, to accommodate variable renewable generation, resource adequacy must consider system flexibility, i.e., sophisticated operating strategies must be established to fully utilize the system resources. Especially, in the follow-up related NERC's reports, a set of 'flexibility requirements' for system operation has been identified [2] [3]. The 'flexibility requirements' include ramping requirements, minimum generation levels, required shorter scheduling intervals, transmission interconnections, etc.

Identifying flexibility resources and constructing methods to fully use them are critical for accommodating large-scale renewables. Establishing measures is a prerequisite for implementing this target. NERC's Reliability Metrics Working Group claimed that the traditional reliability concept needs to be updated to accommodate variable resources. Three categories of risks, i.e., condition- driven risk, event-driven risk, and standard/status-driven risk [4], have been proposed to assess a system operating risks. The condition-driven risk indictors identify factors that positively or negatively impact operating reliability. NERC defined that

A collection of these indicators measures how far reliability performance is from desired outcome, and if the performance is headed in the preferred direction.

Condition-driven risk (CDR) indicates the operating reliability under a given operation condition and the tendency of the system's variables. For a system with large-scale renewables, except for the traditional risk sources, such as loss of components, the risk associated with variable injections should be highlighted. As a result, sophisticated conditiondriven risk indictors should be constructed. A comprehensive CDR indictor should have the capability to describe the essential uncertain of injections, as well as the flexibility provided by other system components.

The objective of this paper is to propose a condition-driven indictor to estimate the expected time for a given operating

This research is partly supported by National Key Research Program(973 Program, 2012CB215102), National Natural Science Foundation of China (51277155), Research Grant Council of Hong Kong SAR (GRF 7124/11E and ECS739713), and Key Breakthrough Project in 'Guangdong-HongkongMacao'(2010A090602004) 
point leaving the security region due to the predictable tendency and stochastic errors of renewables. Based on the flexibility requirements of the controllable resources defined by NERC, the controllable region of the system is identified with DC power flow based security region first. With the stochastic differential equations based models, the characteristics renewables is described. And then, a conservative estimation of the critical time for the system stay in the controllable region is computed.

\section{PROBLEM FORMULATION}

The proposed critical time is a conservative estimation that the operating point of a power system leaves the steady-state security region due to the uncontrollable injections of renewables. For the computability consideration, in this work, the convex DC power flow based steady-state security region is employed. The injections are classified into two groups, i.e., controllable and uncontrollable injections. The critical time is to estimate the longest time that the operating point can stays in the security region by controlling the controllable injections, i.e., controllable generating units and dispatchable loads. The renewables are modeled as uncontrollable injections with a combination of predictable deterministic components and stochastic errors. Mathematically, stochastic differential equations are used. The stochastic loads can also be involved with the proposed method. Finally, the expected exit-time, i.e., the critical time, can be estimated. The proposed critical time measures the risk of a give operating point associated with the characteristics of the system and renewables. As a result, it is a condition-driven risk indictor.

To solve the proposed model, following three inter-related components are involved.

- identifying controllable region for variable generations based on the controllability of the resources in the system,

- estimating tolerance of variable injections' movements from the given operating points in the identified controllable region, and

- computing the critical time that the variable generations exceed the estimated tolerance according to the characteristics of variable generations.

\section{A. Controllable region}

In this work, the steady-state security constraints, i.e., power flow related constraints, are involved. The controllable region, $\boldsymbol{\Omega}_{\mathbf{C R}}$, is constructed with the flexibility required by NERC. $\boldsymbol{\Omega}_{\mathbf{C R}}$ is a subset of steady-state security region, $\boldsymbol{\Omega}_{\mathbf{S S R}}$. According to the pioneer work [5] [6] [7], the $\boldsymbol{\Omega}_{\mathbf{S S R}}$ is defined on the 'injection space', i.e., the space spanned by the vector of all injections. As a result, $\boldsymbol{\Omega}_{\mathrm{SSR}}$ is independent with the injection and only determined by the system's connection and parameters. In this paper, only the DC power flow based $\boldsymbol{\Omega}_{\text {SSR }}$ is employed. In fact, for a transmission system with largescale wind farms integration, the accuracy of DC power flow is acceptable[6]. This renewable's (especially for wind power) utilization strategy has been widely used in Texas and China[8].

Let $\mathbf{P}$ be the injection vector of real power. Without loss of generality, $\mathbf{P}$ is re-ordered as

$$
\mathbf{P}=\left[P_{\text {Ref }}, \mathbf{P}_{\mathbf{C}}, \mathbf{P}_{\mathrm{SR}}\right]^{T}
$$

where $P_{\text {Ref }}$ is the injection of the reference bus, $\mathbf{P}_{\mathbf{C}}$ is the vector of controllable resources, and $\mathbf{P}_{\mathbf{S R}}$ is vector of renewable outputs, respectively. In this vector, the constant loads are included in $\mathbf{P}_{\mathbf{C}}$ with the same lower and upper limits; meanwhile, controllable generators are described by $P_{\text {Ref }}$ and $\mathbf{P}_{\mathbf{C}}$ with different lower and upper limits. As a result, by giving different limits, all loads and controllable generators can be described by $\mathbf{P}_{\mathbf{C R}}=\left[P_{\text {Ref }}, \mathbf{P}_{\mathbf{C}}\right]^{T}$. The associated bus voltage angles' vector is

$$
\boldsymbol{\theta}=\left[\theta_{\text {Ref }}, \boldsymbol{\theta}_{\mathrm{C}}, \boldsymbol{\theta}_{\mathrm{SR}}\right]^{T}
$$

where $\theta_{\text {Ref }}=0$. Therefore, DC power flow satisfies:

$$
\left[\begin{array}{l}
\mathbf{P}_{\mathrm{C}} \\
\mathbf{P}_{\mathrm{SR}}
\end{array}\right]=\mathbf{B}\left[\begin{array}{l}
\boldsymbol{\theta}_{\mathrm{C}} \\
\boldsymbol{\theta}_{\mathrm{SR}}
\end{array}\right]
$$

where $\mathbf{B}$ is the admittance matrix with resistances are equal to zero. Without reference bus, $\mathbf{B}$ is full rank. By inversing $\mathbf{B}$ and adding the reference bus, (3) can be written as

$$
\left[\begin{array}{l}
\boldsymbol{\theta}_{R e f} \\
\boldsymbol{\theta}_{\mathbf{C}} \\
\boldsymbol{\theta}_{\mathbf{S R}}
\end{array}\right]=\left[\begin{array}{cc}
0 & \mathbf{0} \\
\mathbf{0} & \mathbf{B}^{-1}
\end{array}\right]\left[\begin{array}{l}
P_{R e f} \\
\mathbf{P}_{\mathbf{C}} \\
\mathbf{P}_{\mathbf{S R}}
\end{array}\right]
$$

Let $\mathbf{T}=\left[\begin{array}{cc}0 & \mathbf{0} \\ \mathbf{0} & \mathbf{B}^{-1}\end{array}\right]$, (4) can be re-written in a compact form.

$$
\boldsymbol{\theta}=\mathbf{T P}
$$

Since the power flow on line $s-r$ may be calculated as

$$
P_{L(s-r)}=\frac{1}{x_{s-r}}\left(\theta_{s}-\theta_{r}\right)
$$

where $x_{s-r}$ is the reactance of line $s-r$, (6) can be written in following matrix form.

$$
\mathbf{P}_{\mathbf{L}}=\mathbf{A} \boldsymbol{\theta}
$$

where $\mathbf{P}_{\mathbf{L}}$ is the vector of power flow on transmission lines. By combining (5) and(7), we have

$$
\mathbf{P}_{\mathbf{L}}=\mathbf{A} \cdot \mathbf{T} \cdot \mathbf{P}
$$

or

$$
\mathbf{P}_{\mathbf{L}}=\boldsymbol{\Phi} \cdot \mathbf{P}
$$

where $\boldsymbol{\Phi}=\mathbf{A} \cdot \mathbf{T}$.

The DC power flow based steady-state security region $\mathbf{\Omega}_{\mathrm{SSR}}$ can be defined as follows:

$$
\mathbf{\Omega}_{\mathrm{SSR}}=\left\{\mathbf{P} \mid \mathbf{P}_{\mathbf{L}}^{\min } \leq \boldsymbol{\Phi} \cdot \mathbf{P} \leq \mathbf{P}_{\mathbf{L}}^{\max }, \mathbf{P}^{\min } \leq \mathbf{P} \leq \mathbf{P}^{\max }, \sum_{P_{i} \in \mathbf{P}} P_{i}=0\right\}
$$

For the $\boldsymbol{\Omega}_{\text {SSR }}$ defined by (10), C.-C. Liu have proved that it is a convex polyhedron [6]. All 'flexibility requirements' described in the NERC's reports can be included in the model described by (10). For a given scheduling interval, the 
ramping requirements, minimum generation levels, can be described by the limits of injection vector $\mathbf{P}$; meanwhile, the transmission interconnections can be described by DC power flow equations and their limits.

The 'controllable region', $\boldsymbol{\Omega}_{\mathbf{C R}}$, is on the space spanned by the uncontrollable vector, $\mathbf{P}_{\mathbf{S R}}$. It defined as:

Definition 1: the $\boldsymbol{\Omega}_{\mathrm{CR}}$ is a controllable region on the space spanned by uncontrollable vector, $\mathbf{P}_{\mathbf{S R}}$, if $\forall \mathbf{P}_{\mathbf{S R}}^{*} \in \mathbf{\Omega}_{\mathbf{C R}}$, $\exists \mathbf{P}_{\mathbf{C R}}^{*}=\left[P_{R e f}^{*}, \mathbf{P}_{\mathbf{C}}^{*}\right]^{T}$, satisfies $\left[\mathbf{P}_{\mathbf{C R}}^{*}, \mathbf{P}_{\mathbf{S R}}^{*}\right]^{T} \in \mathbf{\Omega}_{\mathrm{SSR}}$, where $\boldsymbol{\Omega}_{\mathbf{S S R}}$ is the steady-state security region defined by (10).

According to the Definition 1, defined on the space spanned by $\mathbf{P}_{\mathrm{SR}}, \boldsymbol{\Omega}_{\mathrm{CR}}$ is a projection of a convex polyhedron $\boldsymbol{\Omega}_{\mathbf{S S R}}$. Physical meaning of $\boldsymbol{\Omega}_{\mathbf{C R}}$ is that for all uncontrollable injections (such as wind power, within the controllable region, $\boldsymbol{\Omega}_{\mathbf{C R}}$ ), the system can ensure the operating point within the steady-state security region by its controllable resources $\mathbf{P}_{\mathbf{C R}}=\left[P_{R e f}, \mathbf{P}_{\mathbf{C}}\right]^{T}$.

Generally, due to (10), the sum of injections is zero. Therefore, $\boldsymbol{\Omega}_{\text {SSR }}$ is a convex polyhedron on a hyperplane passing through the origin. Furthermore, since the projection is a linear operator, we have the following theorem.

Theorem 1: the controllable region $\boldsymbol{\Omega}_{\mathbf{C R}}$ is a convex polyhedron defined on the space spanned by the uncontrollable injection vector $\mathbf{P}_{\mathbf{S R}}$.

\section{B. Tolerance of uncontrollabe injections}

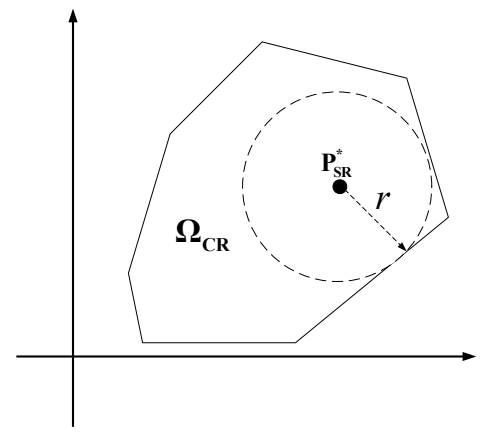

Fig. 1 Distance for given operating point to the boundary of $\mathbf{\Omega}_{\mathbf{C R}}$

The objective is to estimate the distance from the given operating point to the boundary of $\boldsymbol{\Omega}_{\mathbf{C R}}$ for each uncontrollable injection within $\mathbf{P}_{\mathbf{S R}}$. This distance will serve as a parameter to estimate the critical time that the $\mathbf{P}_{\mathbf{S R}}$ leaves $\boldsymbol{\Omega}_{\mathbf{C R}}$. A conservative estimation can be constructing by finding a ball with the largest radius, $r$, within the $\boldsymbol{\Omega}_{\mathbf{C R}}$. The center of the ball is the given operating point of the uncontrollable injection vector, $\mathbf{P}^{*}$ SR. Mathematically, $r$ can be found by solving the following optimization problem.

$$
\begin{array}{ll}
\max & r \\
\text { s.t. } & \left\|\mathbf{P}_{\mathbf{S R}}-\mathbf{P}_{\mathbf{S R}}^{*}\right\|_{2} \leq r, \quad \forall \mathbf{P}_{\mathbf{S R}} \in \mathbf{\Omega}_{\mathbf{C R}}
\end{array}
$$

where \|\|$_{2}$ is the Euclidean norm. According to the Theorem 1, $\boldsymbol{\Omega}_{\mathbf{C R}}$ is a convex polyhedron. (11) is a linear optimization problem.

\section{Critical time}

According to characteristics of uncontrollable injections (renewable outputs) and the tolerance $(r)$, the time that renewable outputs leaving $\boldsymbol{\Omega}_{\mathbf{C R}}$ will be estimated.

As a common practice in the literature, the actual output of a wind generator can be modeled as a forecast value plus a statistical forecast error. Many researches have shown that the standard deviation of forecast error is against the forecast horizon. This work considers the wind farm with many installed wind generators. The forecast error of hourly output of each wind generators is completely correlated for their same geographical locations. Thus, the forecast error of whole wind farm can be the algebraic summation of errors of the connected wind generators.

For the short-term prediction of the wind power, the forecast error is usually assumed as a random variable that obeys the law of Gaussian distribution or truncated Gaussian distribution. [9] has indicated that the expected standard deviation of the wind power in each time period can be estimated, assuming a Gaussian distribution for the next $48 \mathrm{~h}$ with reasonable accuracy. Despite the specific cases, the normally-distributed forecast error for wind power generation is general enough to be applicable with any forecast error probability distribution [10]. Based on [9] [10], [11] has shown the prediction error is normal-like distribution and has given numerous analysis in detail. [12] has indicated that both the prediction errors for load and wind generation are normal distribution. Moreover, the assumption that wind speed forecast error is likely normal distributed is valid as the forecast error is more or less characterized as random process [13], and [14] has suggested that the evolution of the two electrical demand and wind generation is modeled as two independent random processes. The marginal distribution for each of these variables at any time step is assumed to be a normal distribution. Besides, in some other cases, the prediction error is regarded as truncated normal distribution. [15] has presented the distribution of hour ahead and real time forecast errors is an unbiased truncated normal distribution with significant autocorrelation between the subsequent forecasts. [16] has used the truncated normal distribution for analysis. [17] has adopted the truncated normal distribution for comparison among different distribution of prediction error. The assumption in [16]- [18] is supported by the data from [18].

This work focuses on assessment of the short-term operating risk. The actual output of a wind farm is formulated as the forecast value plus a statistical forecast error. Within $\boldsymbol{\Omega}_{\mathbf{C R}}$, each renewable's stochastic output, $\widetilde{P}_{i}(t)$, is described by a stochastic differential equation as follows:

$$
d \widetilde{P}_{i}(t)=u d t+\sigma d \widetilde{B}_{t}
$$

where $u, \sigma$ are two constants, $\widetilde{B}_{t}$ is a standard Brownian motion. On the right side of (12), the first term describes the deterministic component of renewable's prediction and the second term describes the stochastic error.

According to Ito lemma [19], the analytical solution of (12) may be written as 


$$
\widetilde{P}_{i}(t)=u t+\sigma \widetilde{B}_{t}
$$

It is a Brownian motion with drift. For a given time $t$, the mean and variance are

$$
\begin{gathered}
\mathrm{E}\left[\widetilde{P}_{i}(t)\right]=u t \\
\operatorname{Var}\left[\widetilde{P}_{i}(t)\right]=\sigma^{2} t
\end{gathered}
$$

This work will estimate the first time that an uncontrollable injection leaves controllable region. Mathematically, the exittime or the first hitting time can be written as follows.

$$
\xi=\inf \left\{t>0: \exists \widetilde{P}_{i}(t) \notin \boldsymbol{\Omega}_{\mathrm{CR}}\right\}
$$

\section{SOULTION OUTLINE}

The methods to find the critical time, $\xi$, will be proposed in this section.

\section{A. Constructing the controllable region}

The controllable region $\boldsymbol{\Omega}_{\mathbf{C R}}$ is a convex polyhedron by projecting $\boldsymbol{\Omega}_{\text {SSR }}$ to the space spanned by $\mathbf{P}_{\text {SR. The Fourier- }}$ Motzkin elimination [20] is employed to construct $\boldsymbol{\Omega}_{\mathbf{C R}}$.

Without loss of generality, let the dimension of the injection $\mathbf{P}=\left[P_{\text {Ref }}, \mathbf{P}_{\mathbf{C}}, \mathbf{P}_{\mathrm{SR}}\right]^{T}$ is $n$ and the dimension of uncontrollable injections $\mathbf{P}_{\mathbf{S R}}$ is $d$. The projection mapping $\pi: \Re^{n} \mapsto \mathfrak{R}^{d}$ projects $\mathbf{P}=\left[P_{R e f}, \mathbf{P}_{\mathbf{C}}, \mathbf{P}_{\mathbf{S R}}\right]^{T}$ onto its last $d$ dimensions. Since $\boldsymbol{\Omega}_{\mathrm{SSR}}$ is a polyhedron, it can be written in terms of a set of following linear inequality constraints.

$$
\sum_{j=1}^{n} a_{i j} P_{j} \leq b_{i}, \quad i=1, \ldots, m .
$$

The following Fourier-Motzkin elimination algorithm will be used to eliminate one dimension $P_{n}$.

Step 1: Rewrite each constraint $\sum_{j=1}^{n} a_{i j} P_{j} \leq b_{i}$ in the form

$$
a_{i n} P_{n} \leq-\sum_{j=1}^{n-1} a_{i j} P_{j}+b_{i}, \quad i=1, \ldots, m .
$$

If $a_{i n} \neq 0$, divide the both sides by $a_{i n}$. By letting $\overline{\mathbf{P}}=\left(P_{1}, \ldots, P_{n-1}\right)$, we obtain an equivalent representation of $P$ involving the following constraints:

$$
\begin{gathered}
P_{n} \leq d_{i}+f_{i}^{\prime} \overline{\mathbf{P}}, \quad \text { if } a_{i n}>0 \\
d_{j}+f_{i}^{\prime} \overline{\mathbf{P}} \leq P_{n}, \quad \text { if } a_{i n}<0 \\
0 \leq d_{k}+f_{k}^{\prime} \overline{\mathbf{P}}, \quad \text { if } a_{k n}=0
\end{gathered}
$$

Here, each $d_{i}, d_{j}, d_{k}$ is a scalar, and each $f_{i}, f_{j}, f_{k}$ is a vector in $\Re^{n-1}$.

Step 2: Let $Q$ be the polyhedron in $\Re^{n-1}$ defined by the constraints

$$
\begin{aligned}
d_{j}+f_{j}^{\prime} \overline{\mathbf{P}} & \leq d_{i}+f_{i}^{\prime} \overline{\mathbf{P}}, \quad \text { if } a_{i n}>0 \text { and } a_{j n}<0 \\
0 & \leq d_{k}+f_{k}^{\prime} \overline{\mathbf{P}}, \quad \text { if } a_{k n}=0 .
\end{aligned}
$$

By using Fourier-Motzkin elimination algorithm $d-n$ times, the $\boldsymbol{\Omega}_{\mathbf{C R}}$ is constructed.

B. Estimating the distance from a given operating point to the bounday of the controllable region

After implementing Fourier-Motzkin elimination, the controllable region $\boldsymbol{\Omega}_{\mathbf{C R}}$ defined by $m$ linear inequality constraints on $d$ dimensions is identified as follows:

$$
\sum_{j=1}^{d} a_{i j} P_{j} \leq b_{i}, \quad i=1, \ldots, m
$$

For a given operating point of the uncontrollable vector $\mathbf{P}_{\mathrm{SR}}^{*}=\left[P_{1}^{*}, P_{2}^{*}, \cdots, P_{d}^{*}\right]^{T}$, the maximal distance $r$ defined by (11) can be calculated by solving the following Chebychev center problem [21].

$$
\begin{aligned}
& \max r \\
& \text { s.t. }\left\|\left[a_{i 1}, a_{i 2}, \cdots, a_{i d}\right]^{T}\right\|_{2} \cdot r+\sum_{j=1}^{d} a_{i j} P_{j}^{*} \leq b_{i}, \quad i=1, \ldots, m
\end{aligned}
$$

For large-scale problems, the optimization problem defined by (25) can be solved with CPLEX efficiently.

\section{Estimating the distribiution of the critical time}

The renewable output on each dimension is a Brownian motion with positive drift with coefficient $u$ and variance parameter $\sigma^{2}$. The first hitting time is

$$
\xi=\inf \left\{t>0: \widetilde{P}_{i}(t)=r \text { for all } \widetilde{P}_{i}\left(t_{0}\right) \in \mathbf{P}_{\mathrm{SR}}\right\}
$$

where $r$ is the distance identified by (25). The $r$ is a conservative estimation of the distance from a given operating point to the boundary of the controllable region. Since (25) uses a ball to estimate the distance, the distances on all dimensions are the same and equal to $r$. As a result, for each dimension of uncontrollable injection, $\widetilde{P}_{i}(t)$, is equal to the same distance, $r$.

For a uncontrollable injection, $\widetilde{P}_{i}(t)$, according to the characteristics of solution described by (14) and (15), we obtain $\xi=\inf \left\{t: \widetilde{P}_{i}(t)=r\right\}=\inf \left\{t: \widetilde{B}_{t}=\frac{\widetilde{P}_{i}(t)-u t}{\sigma}\right\}$. By the Martingale stopping theorem[22], we have

$$
E\left[\widetilde{B}_{t}(\zeta)\right]=E\left[\widetilde{B}_{t}(0)\right]=0
$$

Furthermore, $\widetilde{B}_{t}(\zeta)=\frac{r-u t}{\sigma}$, therefore,

$$
E\left[\frac{r-u \zeta}{\sigma}\right]=0
$$

or

$$
E[\zeta]=r / u \text {. }
$$

Furthermore, by the similar method, the variance can be calculated as $\operatorname{Var}[\zeta]=r \sigma^{2} / u^{3}$. To sum up, we obtain the Theorem 2. 
Theorem 2: for a system with renewable outputs described by (12), the mean and variance of the critical time defined by (26) are

$$
\mathrm{E}[\zeta]=r / u
$$

and

$$
\operatorname{Var}[\zeta]=r \sigma^{2} / u^{3}
$$

The proposed solution method is illustrated as the following flow chart.

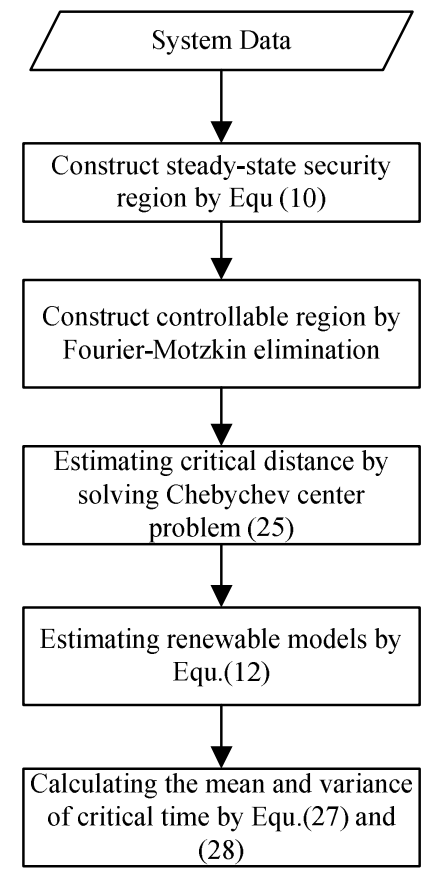

Fig. 2. Flow chart of the proposed method

\section{ILLUSTRATIVE EXAMPLE}

An illustrative example demonstrates the proposed methods. This case studies a system with 3 buses and 3 transmission lines. It is assumed that the admittance of each line is 1 p.u., the lower limit and upper limit of each line are -1 p.u. and 1 p.u., respectively. The lower limit and upper limit of each injection are -10 p.u. and 10 p.u., respectively. Bus 3 is controllable, while Bus 1 and Bus 2 are uncontrollable.

Based on the model proposed in this paper, the steady-state security region $\mathbf{\Omega}_{\mathrm{SSR}}$ is identified first.

$\mathbf{\Omega}_{\mathrm{SSR}}=\left\{\mathbf{P} \mid\left[\begin{array}{c}-1 \\ -1 \\ -1 \\ 0\end{array}\right] \leq \frac{1}{3}\left[\begin{array}{ccc}1 & -1 & 0 \\ 1 & 2 & 0 \\ 2 & 1 & 0 \\ 1 & 1 & 1\end{array}\right]\left[\begin{array}{l}P_{1} \\ P_{2} \\ P_{3}\end{array}\right] \leq\left[\begin{array}{l}1 \\ 1 \\ 1 \\ 0\end{array}\right],\left[\begin{array}{l}-10 \\ -10 \\ -10\end{array}\right] \leq\left[\begin{array}{l}P_{1} \\ P_{2} \\ P_{3}\end{array}\right] \leq\left[\begin{array}{c}10 \\ 10 \\ 10\end{array}\right]\right\}$

The controllable region $\boldsymbol{\Omega}_{\mathbf{C R}}$ on the space spanned by $\left[P_{1}, P_{2}\right]^{\mathrm{T}}$ can be derived analytically as follows:

$$
\left[\begin{array}{l}
-1 \\
-1
\end{array}\right] \leq \frac{1}{3}\left[\begin{array}{cc}
1 & -1 \\
1 & 2 \\
2 & 1
\end{array}\right]\left[\begin{array}{l}
P_{1} \\
P_{2}
\end{array}\right] \leq\left[\begin{array}{l}
1 \\
1
\end{array}\right]
$$

By Fourier-Motzkin elimination described by (17) - (23), the controllable region $\boldsymbol{\Omega}_{\mathbf{C R}}$ can be constructed on the space spanned by $\left[P_{1}, P_{2}\right]^{\mathrm{T}}$. The simulation result is shown in Fig. 4.

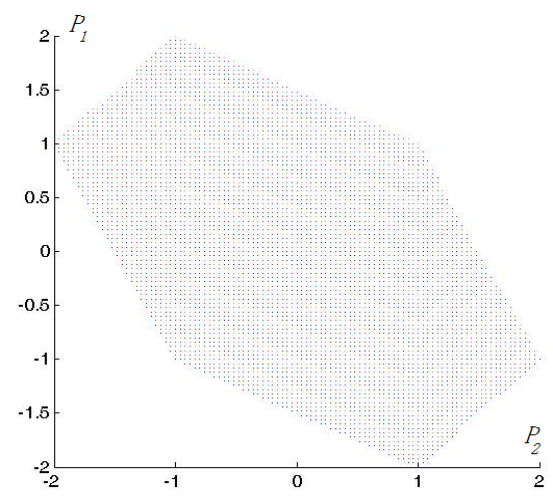

Fig.3. Controllable region $\boldsymbol{\Omega}_{\mathbf{C R}}$ of the 3-bus system identified by FourierMotzkin elimination

The region shown in Fig.4 is the same as the region described by (29). Furthermore, due to the symmetrical characteristic of this system, projection of $\boldsymbol{\Omega}_{\mathrm{SSR}}$ on spaces spanned by $\left[P_{1}, P_{2}\right]^{\mathrm{T}},\left[P_{2}, P_{3}\right]^{\mathrm{T}}$ or $\left[P_{1}, P_{3}\right]^{\mathrm{T}}$ are all with the same shape.

Let the current operating point $\left[P_{1}, P_{2}\right]^{\mathrm{T}}=[0,0]^{\mathrm{T}}$. By solving Chebychev center problem described by (25) with CPLEX, the distance $r=1.3416$. From (29), the distance can be calculated analytically as:

$$
r^{\prime}=\min \left\{\frac{3}{\sqrt{1^{2}+(-1)^{2}}}, \frac{3}{\sqrt{2^{2}+1^{2}}}, \frac{3}{\sqrt{1^{2}+2^{2}}}\right\}=1.3416
$$

The distances calculated by different methods are the same.

Assume the uncontrollable injection $P_{1}$ and $P_{2}$ can be described by following stochastic differential equations.

$$
\begin{gathered}
d \widetilde{P}_{1}(t)=0.35 d t+0.5 d \widetilde{B}_{t} \\
d \widetilde{P}_{2}(t)=0.15 d t+0.75 d \widetilde{B}_{t}
\end{gathered}
$$

Two sampling paths for $P_{1}$ and $P_{2}$ are shown in Fig. 5.

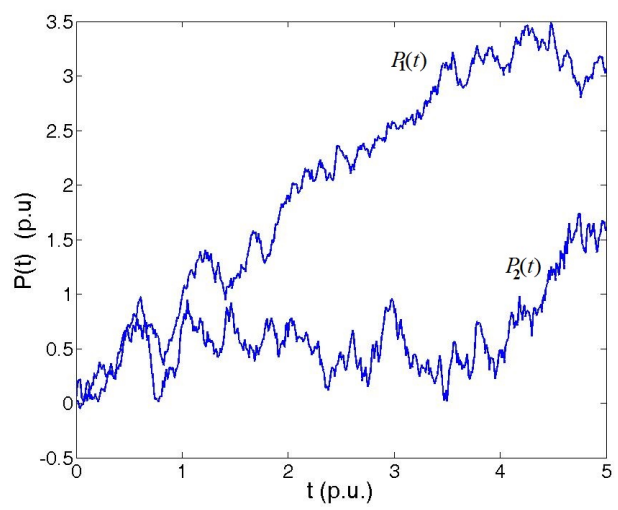

Fig.4. Sampling paths for $P_{l}$ and $P_{2}$

According to (27) and (28), the critical time or first hitting time for $P_{1}$ and $P_{2}$ are: 


$$
\begin{aligned}
& E\left[\zeta_{1}\right]=r / u=1.3416 / 0.35=3.833 \\
& \operatorname{Var}\left[\zeta_{1}\right]=r \sigma^{2} / u^{3}=1.3416 \cdot 0.5^{2} / 0.35^{3}=7.823 \\
& E\left[\zeta_{2}\right]=r / u=1.3416 / 0.15=8.944 \\
& \operatorname{Var}\left[\zeta_{2}\right]=r \sigma^{2} / u^{3}=1.3416 \cdot 0.75^{2} / 0.15^{3}=223.6
\end{aligned}
$$

By 10,000 times Monte Carlo simulations, mean and variance of the critical time for $P_{1}$ and $P_{2}$ are:

$$
\begin{gathered}
\widehat{E\left[\zeta_{1}\right]}=3.8631, \widehat{\operatorname{Var}\left[\zeta_{2}\right]}=7.4339 \text {, and } \\
\widehat{E\left[\zeta_{2}\right]}=8.829, \widehat{\operatorname{Var}\left[\zeta_{2}\right]}=199.8846
\end{gathered}
$$

The simulation results are very close to the results provided by (27) and (28).

In this case, all proposed methods are validated by analytical solutions or simulations. However, for large-scale system, it is difficult to obtain the analytical solutions or very time consuming to implement simulation.

\section{CONCLUSIONS}

Risk assessment is critical for power systems with largescale renewables integration. In this paper, a condition-driven risk indictor, which describes the time that uncontrollable renewable output may leave the security region, is proposed. A systematic method with a set of sophisticated algorithms is proposed to estimate the characteristics of the critical time that the renewable outputs may leave the security region. In the proposed model, all NERC's flexibility requirements are involved. It is believed that the proposed critical time can be employed to construct the condition-driven risk indicators.

By employing the concept of steady-state security region, the proposed methods include: constructing uncontrollable region with Fourier-Motzkin elimination, estimating critical distance with Chebychev center problem, and calculating the characteristics of stochastic critical time with Martingale stopping theorem. An illustrative example validates the proposed method. Due to the high efficiency of the proposed methods, it can be used in the problems on large-scale systems.

\section{ACKNOWLEDGMENT}

The authors would like to thank the reviewers for their valuable comments that have helped to improve the contents and readability of this paper. The authors also would like to thank Professor Felix F. Wu with UC Berkeley for the valuable discussion.

\section{REFERENCES}

[1] NERC. NERC Special Report: Accommodating High Levels of Variable Generation,2009 [Online]. Available: www.nerc.com

[2] NERC. Special Report: Potential Reliability Impacts of Emerging Flexible Resources, 2010 [Online]. Available: http://www.nerc.com/
[3] NERC. Flexibility Requirements and Metrics for Variable Generation: Implications for System Planning Studies, 2010 [Online]. Available: http://www.nerc.com/

[4] NERC. Reliability Metrics Working Group: Integrated Bulk Power System Risk Assessment Concepts, 2010 [Online]. Available: http://www.nerc.com/filez/rmwg.html

[5] F. Wu and S. Kumagai, "Steady-State Security Regions of Power Systems," Circuits and Systems, IEEE Transactions on, vol. 29, pp. 703-711, 1982.

[6] C.-C. Liu, "A New Method for the Construction of Maximal SteadyState Security Regions of Power Systems," Power Systems, IEEE Transactions on, vol. 1, pp. 19-26, 1986.

[7] F. F. Wu, Y. K. Tsai, and Y. X. Yu, "Probabilistic steady-state and dynamic security assessment," Power Systems, IEEE Transactions on, vol. 3, pp. 1-9, 1988.

[8] Y. Hou and J. Zhong, "Challenges Ahead: Currents Status and Future Prospects for Chinese Energy," Power and Energy Magazine, IEEE, vol. 10, pp. 38-47, 2012.

[9] F. Bouffard and F. D. Galiana, "Stochastic security for operations planning with significant wind power generation," in Power and Energy Society General Meeting - Conversion and Delivery of Electrical Energy in the 21st Century, 2008 IEEE, 2008, pp. 1-11.

[10] E. D. Castronuovo and J. A. Peas Lopes, "On the optimization of the daily operation of a wind-hydro power plant," Power Systems, IEEE Transactions on, vol. 19, pp. 1599-1606, 2004.

[11] R. Doherty and M. O'Malley, "A new approach to quantify reserve demand in systems with significant installed wind capacity," Power Systems, IEEE Transactions on, vol. 20, pp. 587-595, 2005.

[12] Y. V. Makarov, C. Loutan, M. Jian, and P. de Mello, "Operational Impacts of Wind Generation on California Power Systems," Power Systems, IEEE Transactions on, vol. 24, pp. 1039-1050, 2009.

[13] Y. V. Makarov, C. Loutan, M. Jian, P. de Mello, and L. Shuai, "Impacts of wind generation on regulation and load following requirements in the California system," in Power and Energy Society General Meeting - Conversion and Delivery of Electrical Energy in the 21st Century, 2008 IEEE, 2008, pp. 1-9.

[14] K. Methaprayoon, W. J. Lee, C. Yingvivatanapong, and J. Liao, "An integration of ANN wind power estimation into UC considering the forecasting uncertainty," in Industrial and Commercial Power Systems Technical Conference, 2005 IEEE, 2005, pp. 116-124.

[15] L. Ning, R. Diao, R. P. Hafen, N. Samaan, and Y. V. Makarov, "A comparison of forecast error generators for modeling wind and load uncertainty," in Power and Energy Society General Meeting (PES), 2013 IEEE, 2013, pp. 1-5.

[16] V. S. Pappala, I. Erlich, K. Rohrig, and J. Dobschinski, "A Stochastic Model for the Optimal Operation of a Wind-Thermal Power System," Power Systems, IEEE Transactions on, vol. 24, pp. 940-950, 2009.

[17] A. G. Tsikalakis, Y. A. Katsigiannis, P. S. Georgilakis, and N. D. Hatziargyriou, "Determining and exploiting the distribution function of wind power forecasting error for the economic operation of autonomous power systems," in Power Engineering Society General Meeting, 2006. IEEE, 2006, p. 8 pp.

[18] Members of the Renewables Workgroup "Integration of Renewable Resources: Transmission and operating issues and recommendations for integrating renewable resources on the California ISO-controlled Grid," Califonia Independent System Operator, November 2007.

[19] B. OKsendal, Stochastic Differential Equations, An Introduction with Application, 4ed: Springer-Verlag Heidelberg New York, 1995.

[20] D. Bertsimas and J. N. Tsitsiklis, Introduction to Linear Optimization. Belmont, Massachusetts, USA: Athena Scientific, 1997.

[21] S. Boyd and L. Vandenberghe, Convex Optimization: Cambridge University Press, 2004.

[22] J. M. Steele, Stochastic Calculus and Financial Application. New York, NY, USA: Springer, 2000. 